and Fabry's diseases, as well as metachromatic leucodystrophy).

Leucocyte studies of families have done much to increase our knowledge of the genetics of the inherited metabolic disorders and in many cases the heterozygous state can be clearly delineated using these readily available cells. Direct enzyme assay on the isolated cells is usually satisfactory, although in some cases the white cell of choice may be the stimulated lymphocyte. Accumulation of material within leucocytes (Pompe's disease, cystic fibrosis) may aid diagnosis, as do functional tests on leucocytes, such as the loss of ability of these cells to phagocytose and kill bacteria or to take up nitrotetrazolium blue as is seen in chronic granulomatous disease.

Leucocyte assay (not necessarily enzyme orientated) may also have potential use in early recognition of graft rejection, and radioactive leucocytes may prove useful probes in seeking out septic foci in the body. Preliminary reports suggest that pyruvate kinase levels in the leucocyte of the mother may be a useful diagnostic tool to monitor fetal malnutrition. Histochemical examination of enzyme activity in the leucocyte has been suggested to be of value in the differential diagnosis of certain leukaemias.

\section{Leucocyte Changes in Cirrhosis}

G. E. LEVIN AND D. N. BARON (Department of Chemical Pathology, The Royal Free Hospital, London) Patients with cirrhosis and ascites commonly develop electrolyte disturbances, of which hypokalaemia and hyponatraemia are the most frequently encountered. Few attempts have bsen made to investigate the intracellular water and electrolyte status of such patients, who are often on a low salt diet and taking potassium losing diuretics.

Viable leucocytes were isolated from venous blood by the method of Baron and Ahmed (Clin. Sci., 37, 205, 1969) and the intracellular sodium, potassium, and water contents measured. Nineteen ineasurements were made on 13 patients with cirrhosis and ascites. These results were compared with those obtained from 35 healthy control subjects. There was no evidence of any leucocyte potassium deficiency when the results were expressed in terms of the cell dry weight. There was a significant increase in the cell water to solid ratio, and this was associated with a considerable fall in the cell potassium concentration in the cell water. It was observed that cell water values were within normal limits in all five of the severely hyponatraemic individuals and that in such patients the intracellular sodium and potassium content was considerably reduced in comparison with the other cirrhotic subjects.

\section{Studies of Leucocyte Electrolytes in Renal Failure and Hypertension}

N. F. JONES (St Thomas' Hospital, London) There are considerable difficulties in the study of intracellular electrolytes but it is clearly desirable that we should know much more about the intracellular compartment in disease. The leucocyte is a suitable model for such studies, having the methodological advantages of an 'isolated cell preparation'. Compared with erythrocytes, leucocytes are less uniquely specialized and react more quickly to changes in the extracellular fluid. In this report I describe studies of leucocyte electrolyte composition in chronic renal failure and in hypertension.

Leucocytes from patients with advanced renal failure were swollen cells with an increased water content. The latter correlated better with the cell sodium content, which was elevated, than with cell potassium content in these patients, whereas the converse is true in normal subjects. The cell content of potassium and its concentration in cell water were reduced in uraemia. These findings implied abnormalities of leucocyte cation transport in uraemia, and, in confirmation, we found that the rate constant for active (ouabain-sensitive) sodium efflux from this cell was markedly reduced in chronic renal failure. Passive fluxes of sodium across the cell membrane remained normal.

Observations on patients receiving maintenance haemodialysis and in others with acute renal failure indicate that the abnormality described above may develop rapidly and are also rapidly improved, but not totally corrected, by dialysis.

Examination of the results obtained in uraemia suggested a possible relationship between leucocyte sodium content and the blood pressure. Measurements were therefore made on leucocytes from patients with untreated essential hypertension and normal renal function. Control normotensive subjects were carefully matched for age, sex, and race. Leucocyte sodium and water contents were elevated in essential hypertension, and the rate constant for active sodium efflux was reduced. Whether these findings are a consequence of the hypertensive state or reflect a fundamental abnormality in cell sodium regulation in this condition is the subject of continuing research.

\section{Cytochemistry of Leucocytes}

D. CATovsKy (MRC Leukaemia Unit, Royal Postgraduate Medical School, London) Cytochemical tests applied to leucocytes may help in the diagnosis and classification of leukaemia, particularly the acute types (AL). It is convenient to divide the cytochemical reactions into those which help to characterize myeloid cells and those useful for the study of lymphoid cells.

Myeloid cells: some enzymes are regarded as specific markers of myeloid differentiation.

1. Myeloperoxidase: localized in the early granules of granulocytes and monocytes. The enzyme present in neutrophils is cyanide-sensitive, while part of the one present in eosinophils is cyanide-resistant Results with the peroxidase reaction often parallel those obtained with the Sudan Black B stain for phospholipids The reaction is usually positive in acute myeloid leukaemia and is negative in the lymphoblastic and undifferentiated types of AL.

2. Chloroacetate esterase: probably localized in the same granules as myeloperoxidase; leukaemic eosinophils may be positive while normal ones are not.

3. Non-specific esterase: a peptidase specific to monocytic cells, particularly when the reaction is inhibited by the presence of $\mathrm{NaF}$.

4. Lysozyme: a cytobacterial method could easily be applied for the study of this enzyme in leucocytes.

(3) and (4) are useful in the differential diagnosis of $\mathrm{AL}$; leukaemic monocytes are, as a rule, positive; myeloblasts and lymphoblasts are negative.

5. Alkaline phosphatase: present in mature neutrophils but probably not, as in the rabbit, in their secondary granules. Useful in the differential diagnosis between myeloproliferative disorders and leukaemoid reactions. Its changes in disease may only reflect changes in the life span of neutrophils.

Lymphoid cells: no specific enzymes of lymphocytes have been found. However, a high content in acid phosphatase demonstrated in lysosomal granules and in the membranes of the Golgi apparatus 